\title{
Introductory notes for the Acta IMEKO Special Section on the '2018 IEEE International Workshop on Metrology for Industry 4.0 \& loT' $^{\prime}$
}

\author{
Alessandro Depari, Emiliano Sisinni \\ Department of Information Engineering, University of Brescia, Via Branze 38 - 25123, Brescia (ITALY)
}

\begin{abstract}
Section: Editorial
Citation: Alessandro Depari, Emiliano Sisinni, Introductory notes for the Acta IMEKO Special Section on the '2018 IEEE International Workshop on Metrology for Industry 4.0 \& IoT', Acta IMEKO, vol. 8, no. 2, article 1, June 2019, identifier: IMEKO-ACTA-08 (2019)-02-01
\end{abstract}

Editor: Dušan Agrež, University of Ljubljana, Slovenia

Received June 20, 2019; In final form June 20, 2019; Published June 2019

Copyright: This is an open-access article distributed under the terms of the Creative Commons Attribution 3.0 License, which permits unrestricted use, distribution, and reproduction in any medium, provided the original author and source are credited.

Corresponding authors: Alessandro Depari, e-mail: alessandro.depari@unibs.it; Emiliano Sisinni, e-mail: emiliano.sisinni@unibs.it

\section{Distinguished Readers,}

The here finalized issue of Acta IMEKO presents research works accepted for the special section devoted to the 2018 IEEE International Workshop on Metrology for Industry 4.0 \& IoT (MetroInd4.0\&IoT). The conference was hosted by the University of Brescia, Brescia, Italy, and took place on April 1618. 2018. The workshop aimed to discuss the contributions, the challenges and the new opportunities offered by Industry 4.0 and Internet of Things (IoT) paradigms for the development of new measurement methods and apparatus.

Therefore, MetroInd4.0\&IoT was the ideal place where researchers and practitioners from industry, academia, government and standardization committee can meet to review and discuss most recent trends in the area of interest and share innovative research directions.

The conference participants enjoyed the talks in the general sessions, complemented by those of the several special sessions focused on Perception Methods to Enhance the role of the Man in the Loop, on Embedded vision methods and systems for edgecomputing and IoT applications, on Smart Measurement Systems for on-line Quality Control, on Synchronization for Internet of Things, on Measurement Systems and Approaches for Smart Manufacturing, on Measurement Systems in the Industrial IoT Era, and on Standards and Technologies for Cybersecurity of IoT and Industry 4.0.

Out of the 49 presented papers, from 11 countries, after a review process with highly qualified referees, ten papers have been accepted for this issue. Innovative approaches and application scenarios have been devised by the authors of the selected paper, as briefly resumed in the following.

In the paper "A RAMS analysis for a precision scale-up configuration of the 'Smart Street' pilot site: an industry 4.0 case study", the authors describe the results of a RAMS analysis in a smart cities scenario (in particular, a smart lighting solution named "Smart street"). They combined Reliability Analysis with Failure Mode, Effects, and Criticality Analysis in order to identify the best configuration allowing to scale up the system without incurring in marginally reliable configurations that instead prove to be extremely vulnerable from the point of view of operational availability.

The work "Applying heuristics to the selection and prioritization of security assessment items in software assessment: the case of ISO/IEC 27001", focuses on security assessment heuristics. The proposed approach (named HCApp$\mathrm{Sec}$ ) is flexible and allows the inclusion of security dimensions and properties. HCApp-Sec is designed to support (i) the generation of high-coverage assessment designs, and (ii) the evaluation of security standards with respect to the coverage of security aspects, as demonstrated analysing assessment items of the well-known security standard ISO/IEC 27001.

The work by Castello et al. deals with measurement equipment based on synchro phasor technology for the monitoring of power systems. In particular, Authors present an innovative Phasor Data Concentrator (PDC) design based on a low-cost platform, to be used coupled with low-cost Phasor Measurement Units (PMUs). The proposed device is characterized through several experimental tests for performance evaluation, in terms of both time synchronization and capability of managing several PMU data streams, as well as evaluating the feasibility of some additional functionalities and control logics under different possible scenarios.

Avallone et al. consider the most relevant physical-layer parameters used by Long Term Evolution (LTE) standard to optimize a Multiple Input Multiple Output (MIMO) system. An urban scenario is considered, taking into account actual signal 
propagation. The analysis of performance, carried out at different frequencies and bandwidths, allows to verify if standard prescriptions are satisfied or not.

Bellitti et al. describe an impedance-based measuring system aiming at monitoring bacterial growth in Petri dishes, while they are inside an incubator of the WASPLab ${ }^{\circledR}$ specimen processing platform. Besides having reconfiguration capabilities, to comply with user necessities, it has additional features that foster its connectivity with the WASPLab ${ }^{\circledR}$ and other devices, developing a structure compliant with the Industry 4.0 model. Experimental results show system correct operation both in an ad hoc setup and while working with the WASPLab®.

The paper by Lamonaca et al. concerns Structural Health Monitoring (SHM), with particular care to the concept of exchanging information among nearby buildings, to increase the timeliness of the alerts due to civil infrastructures failures. The proposed approach is based on the Internet-of-Things paradigm (SHM-IoT) and it carries out both a localized monitoring on a single building (collecting information from several sensors) and a wider monitoring on a group of building. The hardware and software architectures of the proposed SHM-IoT are presented together with the time synchronization requirements; experiments have been performed to validate the proposed approach in real scenarios.

A low power IoT architecture for the monitoring of chemical emissions, to be deployed in industrial plants or public buildings, is presented in the paper by Addabbo et al. Each developed sensor node hosts a broad range of environmental and electrochemical gas sensor. Sensor nodes are complemented with LoRa LPWAN connectivity that allows Wide Area data transmission (about $3 \mathrm{~km}$ ). A multilayer, modular topology has been adopted, combining the features of LoRa technology with shorter and larger range telecommunication channels, resulting in an IoT framework that can be customized according to the physical and technical features of the deployment scenario.

The paper by Angrisani et al., suggests the use of an IoToriented platform, programmed in the LabVIEW environment and leveraging the TCP/IP protocol stack, as a viable and effective solution for implementing an Automated Testing Equipment (ATE). Remote access capability makes the proposed solution an ideal training tool for technicians. In particular, the scalability, complexity, and responsiveness of the developed system have been discussed.

The work "An IoT framework for the assessment of indoor conditions and estimation of occupancy rates: results from a real case study" exploits the heterogeneity of IoT architectures to provide aggregated information from different sensor data. In particular, carbon dioxide concentration is combined with temperature and humidity parameters for indoor air quality (IAQ) evaluation in one of the buildings at the smart campus of the University of Brescia. Additionally, the same sensor data provide reliable information about the presence of students/users, paving the way to customized future services and management optimization procedures.

In the paper "On the performance of OPC UA and MQTT for data exchange between industrial plants and cloud servers", two well-known protocols, widely used in both IoT and Industrial IoT applications, i.e. the open platform communication-unified architecture (OPC UA) and the message queuing telemetry transport (MQTT), are compared. In particular, time-related performance for sending/receiving data to/from cloud servers are analysed. The proposed measurement methodology has been applied to specific use cases, demonstrating that the OPC UA complexity results in a slower data exchange with respect to MQT'T.

We are deeply honoured to give our contribution to the measurement science community and to act as Guest Editors for this innovative and challenging issue of Acta IMEKO. We are delighted to provide the readers around the world the opportunity to access the recent advancements in measurements for IoT and Industry 4.0 scenarios and new opportunities offered by IoT and Industry 4.0 paradigms to instrumentation and measurement research fields. We are looking for new innovations to arise from this scientifically sound basis.

The here presented release is the result of hard but rewarding scientific work, with authors, reviewers, copy-editors, layout editors and, last but not least, the Editor in Chief, Prof. Dušan Agrež, providing his support for all this period.

\section{Alessandro Depari \\ Emiliano Sisinni \\ Guest Editors}

